\title{
Evolución y pronóstico oncológico de fístulas anastomóticas esofágicas en el tratamiento del cáncer de esófago. Estudio comparativo según vía de ascenso de tubo gástrico
}

\author{
Italo Braghetto M. ${ }^{1}$, Dr. Manuel Figueroa G. ${ }^{1}$, Dra. Belén Sanhueza P. ${ }^{1}$, Héctor Valladares H. ${ }^{1}$, \\ Dr. Gonzalo Cardemil H. ${ }^{1}$, EU. Solange Cortés L. ${ }^{1}$, Int. Caterina Contreras B. ${ }^{2}$
}

\section{Outcome and oncological prognosis of esophageal anastomotic fistulas in the treatment of esophageal cancer. Comparative study according to gastric tube ascent}

\begin{abstract}
Introduction: Post operative leaks of esophageal anastomosis after esophagectomy is a risky event associated with poor postoperative evolution. Its frequency and severity will depend mainly on surgical technical aspects. Objectives: To analyze the frequency, management and prognosis of leakage of esophageal anastomosis after esophagectomy for esophageal cancer. Material and Method: Analysis of our prospective oncologic database of patients with esophageal cancers submmitted to esofagectomy. Statistical analysis with Fisher's exact test. Results: 34 out of 37 esophageal cancer patients were included submitted to completely invasive mini esophagectomy. Cervical anastomosis was performed in $79.4 \%$ of patients, in the remaining $20.6 \%$, a distal esophagectomy with intrathoracic anastomosis was performed. The leak rate was 38.2\% (13/34), of these, 69.2\% (9/13) correspond to grade Clavien - Dindo I - II complications. The leak rate was $54.5 \%$ (6/11) for retro-sternal gastric ascensus and $43.7 \%(7 / 16)$ for mediastinal route, without significative difference $(\mathrm{p}=1.0)$. The reoperation rate was $11.7 \%$, being a $100 \%$ secondary to mediastinal ascensus, 3 of them were submitted to thoracoscopic toilets and an innominate vein repair. Postoperative mortality rate was $5.8 \%$, all concentrated in the group of patients with posterior mediastinal ascensus, but without statistical difference $(p=0.26)$. Conclusion: Leaks are frequent in patients operated on for esophageal cancer, especially after cervical esophago-gastro-anastomosis with anterior route for ascensus. However, retro-sternal ascensus did not require re-operations, nor postoperative mortality compared to gastric ascensus through posterior mediastinum

Key words: Esophageal cancer, Esophagectomy, Anastomotic leaks.
\end{abstract}

\section{Resumen}

Introducción: La filtración de anastomosis esofágica es un evento que se asocia a mala evolución postoperatoria $\mathrm{Su}$ frecuencia y gravedad dependerá principalmente de aspectos técnicos quirúrgicos. Objetivos: Analizar la frecuencia, manejo y pronóstico de las filtraciones de anastomosis esofágicas en esofagectomías por cáncer comparando la vía de ascenso del tubo gástrico y sitio de anastomosis. Material y Método: Análisis de base prospectiva de pacientes con cáncer esofágico sometidos a esofagectomía. Análisis estadístico con test exacto de Fisher. Resultados: De un total de 37 pacientes con cáncer esofágico tratados en nuestra institución en el período de estudio ( 5 años), se incluyeron 34 esofagectomías totalmente mini invasivas secundarias a cáncer de esófago. Un 79,4\% correspondieron a esofagectomías totales con anastomosis cervical, en el $20,6 \%$ restante se realizó esofagectomía distal con anastomosis intratorácica. La tasa de filtración de la anastomosis esofágica fue de un 38,2\% (13/34), todas fueron secundarias a esofagectomías totales. De estas un 69,2\% (9/13) se clasificaron como Clavien - Dindo I-II. La tasa de filtración fue de 54,5\% (6/11) para ascenso retroesternal y 43,7\% (7/16) para ascenso mediastínico, $\sin$ ser estadísticamente diferente $(\mathrm{p}=1,0)$. La tasa de reoperaciones fue de un $11,7 \%$, siendo en todas secundario a ascensos mediastínicos posteriores, de estas fueron 3 casos de aseos vídeo-toracoscópicos y una reparación de vena innominada. No existió diferencia estadística entre las vías de ascenso y la tasa de
'Departamento de Cirugía Hospital Clínico de la Universidad de Chile. Santiago, Chile. IInterna de Medicina Universidad de Chile. Facultad de Medicina. Santiago, Chile.

Sin financiamiento Sin conflicto de interés.

Recibido el 15 de mayo de 2017, aceptado para publicación el 1 de agosto de 2017.

Correspondencia a: Italo Braghetto M. ibraghet@hcuch.cl 
reoperaciones $(p=0,26)$. La serie presentó una mortalidad quirúrgica de 5,8\% concentrados todos en el grupo de pacientes con esofagectomías totales con ascenso mediastínico posterior. Conclusión: Las filtraciones en anastomosis esofágicas son frecuentes en pacientes operados con intención curativa de cáncer esofágico. Las filtraciones de anastomosis esofágicas cervicales con ascenso retroesternal no requirieron reoperaciones, ni presentaron mortalidad postoperatoria.

Palabras clave: Cáncer de esófago; Esofagectomía; Filtración de anastomosis.

\section{Introducción}

El tratamiento quirúrgico del cáncer esofágico con intención curativa sigue siendo el Gold Standar. A pesar de los avances en el último tiempo, representa un desafío quirúrgico dado la alta tasa de complicaciones quirúrgicas y mortalidad operatoria ${ }^{1,2}$. La forma de reconstrucción del tránsito intestinal habitualmente es hecha como primera alternativa con una tubulización gástrica; las vías de ascenso más utilizadas de este tubo gástrico son la retroesternal (mediastino anterior) y la transmediastínica posterior. Sin embargo, este enfrentamiento posee una morbilidad quirúrgica y mortalidad considerable, cercanas a $40 \%$ y $7 \%$ respectivamente, siendo las complicaciones respiratorias y la filtración de la anastomosis las complicaciones más frecuentes, asociadas a mortalidad ${ }^{3}$.

La filtración de la anastomosis esofágica es una complicación quirúrgica temida, su frecuencia va a depender de la técnica quirúrgica, la vía de ascenso gástrico y la ubicación de la anastomosis, presentando una tasa entre 3-14\% cuando se efectúa anastomosis intramediastínica y entre $15-60 \%$ cuando se usa la vía retroesternal con anastomosis cervical ${ }^{4}$.

Una vez instaurada, la filtración de anastomosis esofágicas, es un evento postoperatorio que pone en riesgo la evolución y pronóstico del paciente, existiendo reportes de hasta un $20 \%$ de mortalidad en la población afectada dependiendo de la localización y vía de ascenso del tubo gástrico ${ }^{4}$.

El objetivo primario de este trabajo es analizar la frecuencia, manejo de las filtraciones de anastomosis esofágicas en esofagectomías por cáncer comparando vía de ascenso del tubo gástrico y sitio de anastomosis, en los últimos 5 años de nuestro hospital. Un objetivo secundario es determinar posibles factores predictivos de fistulización y si la presencia de fístula esofágica impacta en el pronóstico oncológico alejado.

\section{Material y Método}

Este trabajo es un análisis retrospectivo de la base de datos oncológica prospectiva de nuestra institu- ción. El período de análisis fue entre enero de 2011 y junio de 2016 .

Se revisaron cánceres esofágicos en población adulta, tratados en forma quirúrgica con intención curativa, con ascenso gástrico como método de reconstrucción del tracto digestivo. Para el análisis estadístico las anastomosis se dividieron intratorácicas y cervicales, en estas últimas se subdividieron aquellos pacientes con ascenso gástrico transmediastínico posterior de los retroesternales (mediastino anterior).

Los pacientes excluidos fueron aquellos en los que se usó ascenso de colon, resecciones endoscópicas o procedimientos paliativos.

Los eventos adversos postoperatorios se clasificaron según Clavien-Dindo. La mortalidad quirúrgica se definió desde el momento de la esofagectomía hasta 30 días postoperatorios y la mortalidad hospitalaria hasta los 90 días.

Las descripciones de variables continuas se expresaron empleando mediana y desviación estándar, mientras que las variables categóricas fueron descritas a través de porcentajes.

En relación a la terapia adyuvante, un $64,7 \%$ de nuestra población tenía indicación de quimioradioterapia postoperatoria según la decisión del comité oncológico dado el estadio evolutivo (mayoritariamente en estadio IIIb).

Para el análisis estadístico analítico se empleó el test exacto de Fisher, como límite de significancia se utilizó un $\mathrm{p}<0,05$ con el programa Stata 13.

\section{Resultados}

De un total de 37 pacientes con cáncer esofágico tratados en nuestra institución en el período de estudio (5 años), se incluyeron 34 con esofagectomías mínimamente invasivas para el análisis de los resultados, de estos, $13(38,2 \%)$ presentaron filtración anastomótica. Un $68 \%$ de la muestra fueron hombres ( 23 pacientes), la edad media fue 64 años con un intervalo entre 41 y 83 años. Un $79 \%$ de los pacientes presentaban comorbilidades destacando: 
sobrepeso/obesidad en un 53\%, hipertensión arterial en un $41 \%$, enfermedad por reflujo gastroesofágico patológico/esófago de Barrett en un $26 \%$ y diabetes mellitus en un $18 \%$ de los pacientes. El índice de masa corporal (IMC) medio fue $25,8 \mathrm{~kg} / \mathrm{mt}^{2}$ (1637,4) (Tabla 1).

El $100 \%$ de la muestra fue resecada por vía mini invasiva. Un 19,6\% (7 pacientes) fueron esofagectomías con anastomosis intratorácica, el 79,4\% (27 pacientes) restante fueron anastomosis cervicales, en las cuales un 59,3\% (16 casos) de los tubos gástricos tuvo una vía de ascenso vía mediastino posterior y un 40,7\% (11 casos) retroesternal. En la Figura 1 se muestran los resultados en cuanto a filtración según localización de anastomosis, vía de ascenso de tubo gástrico y número de filtraciones.

La tasa global de filtración anastomótica fue de $38,2 \%$ (13 pacientes), un 69,3\% correspondieron a complicaciones leves según clasificación de Clavien-Dindo, mientras que un 30,7\% fueron complicaciones tipo III-V.

Al discriminar según técnica quirúrgica, las 7 esofagectomías distales con anastomosis intratorácica no presentaron fístula de la anastomosis propiamente tal, sí ocurrió en un paciente que habiendo sido dado de alta en buenas condiciones presentó una filtración de la línea de grapas del tubo gástrico adyacente a la anastomosis en la segunda semana postoperatoria, que requirió manejo protésico con buena evolución final (C-D IIIb).

De los 27 pacientes con anastomosis cervical 48,1\% (13 pacientes) filtraron. Al discriminar la vía de ascenso del tubo gástrico para distintas anastomosis cervicales se obtuvo una tasa de $54,5 \%$ de filtración en ascensos retroesternales y un 43,7\% en ascensos mediastínicos posteriores, sin obtener diferencia estadística entre ambas. En aquellos pacientes con anastomosis cervical con ascenso
Tabla 1. Análisis estadístico de variables demográficas en pacientes agrupados según filtración de anastomosis esofágica

\begin{tabular}{|c|c|c|c|}
\hline Variable analizada & $\begin{array}{l}\text { Sin filtración } \\
\quad(n=21)\end{array}$ & $\begin{array}{l}\text { Con filtración } \\
\quad(n=13)\end{array}$ & $\mathbf{p}$ \\
\hline $\begin{array}{l}\text { Sexo } \\
\text { Hombres } \\
\text { Mujeres }\end{array}$ & $\begin{array}{r}13 \\
8\end{array}$ & $\begin{array}{r}10 \\
3\end{array}$ & 0,465 \\
\hline Edad & $63,1 \pm 9,4$ & $69,8 \pm 9,3$ & 0,052 \\
\hline $\begin{array}{l}\text { Comorbilidades } \\
\text { HTA } \\
\text { DM } \\
\text { Reflujo } \\
\text { Barret } \\
\text { Obesidad } \\
\text { Tabaco }\end{array}$ & $\begin{array}{r}18 \\
8 \\
4 \\
7 \\
7 \\
5 \\
6\end{array}$ & $\begin{array}{l}9 \\
5 \\
2 \\
1 \\
1 \\
2 \\
6\end{array}$ & $\begin{array}{l}0,386 \\
1,000 \\
1,000 \\
0,116 \\
0,116 \\
0,261 \\
0,461\end{array}$ \\
\hline IMC & $27,5 \pm 4,9$ & $25,1 \pm 6,3$ & 0,263 \\
\hline $\begin{array}{l}\text { ASA } \\
\text { I } \\
\text { II } \\
\text { III }\end{array}$ & $\begin{array}{r}4 \\
17 \\
0\end{array}$ & $\begin{array}{l}3 \\
8 \\
2\end{array}$ & 0,202 \\
\hline $\begin{array}{l}\text { Estadiaje } \\
\text { T } \\
1 \\
2 \\
3 \\
4\end{array}$ & $\begin{array}{l}6 \\
7 \\
6 \\
2\end{array}$ & $\begin{array}{l}2 \\
3 \\
8 \\
0\end{array}$ & 0,237 \\
\hline $\begin{array}{r}\mathrm{N} \\
0 \\
1\end{array}$ & $\begin{array}{r}9 \\
12\end{array}$ & $\begin{array}{l}6 \\
7\end{array}$ & 0,851 \\
\hline $\begin{array}{l}\text { Histología } \\
\text { Adenoca } \\
\text { Escamoso } \\
\text { Otro }\end{array}$ & $\begin{array}{r}13 \\
7 \\
1\end{array}$ & $\begin{array}{l}7 \\
6 \\
0\end{array}$ & 0,591 \\
\hline Mortalidad quirúrgica & 0 & 2 & 0,139 \\
\hline Sobrevida a 2 años & $76,9 \%$ & $16,7 \%$ & 0,003 \\
\hline
\end{tabular}

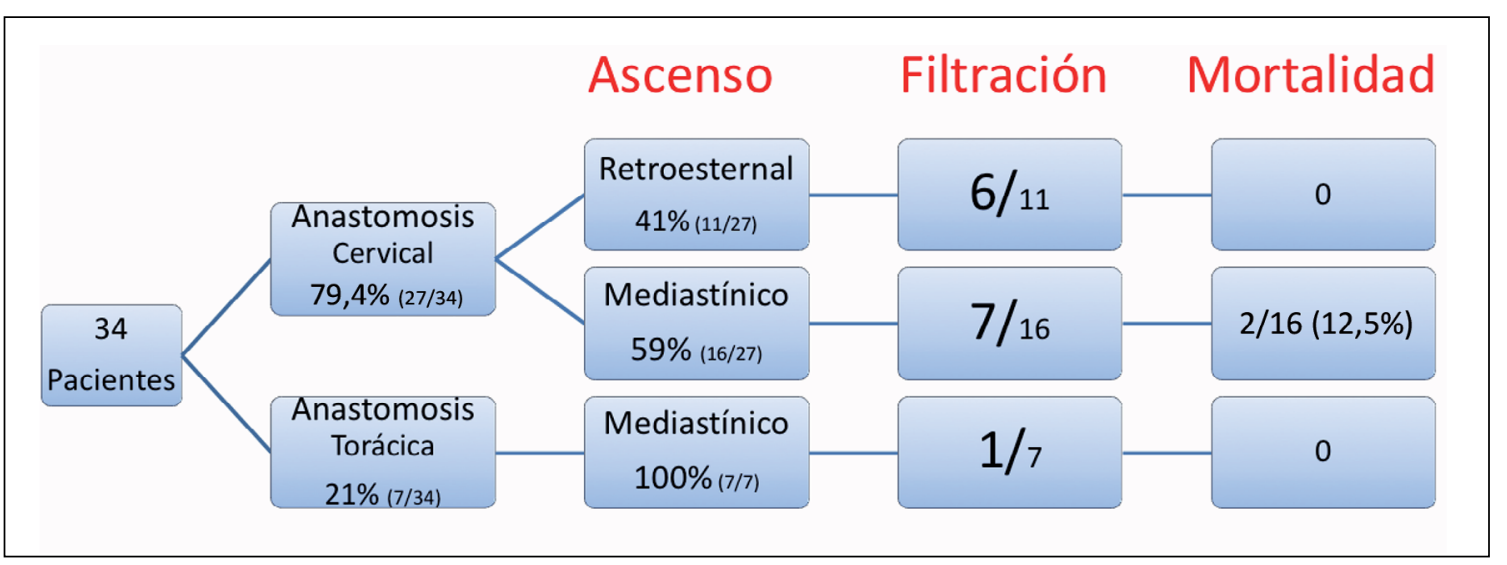


Figura 2. Gráfica de sobrevida Kaplan-Meier (a) global y (b) de población con o sin filtración de anastomosis esófago gástrica. Tiempo expresado en meses.

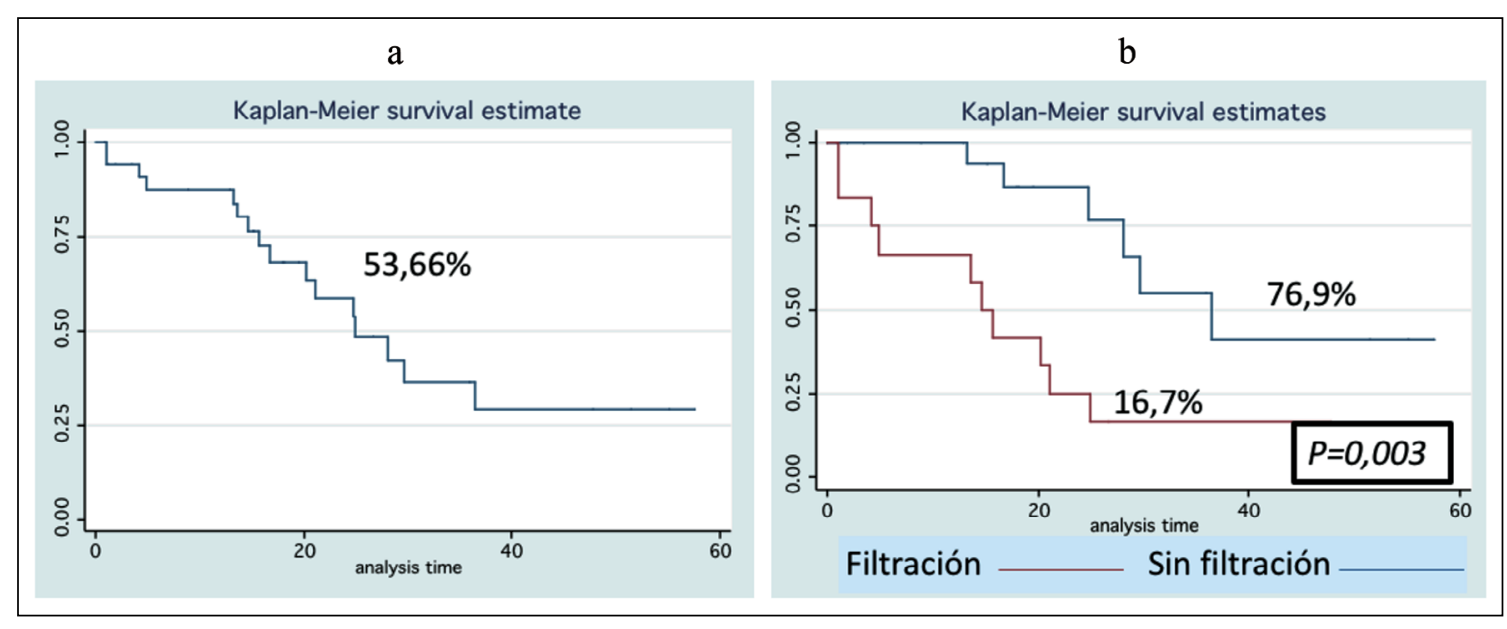

gástrico retroesternal (mediastino anterior) todas las filtraciones fueron C-D I-II, logrando ser manejadas en su totalidad por vía conservadora con curación de cervicotomía y antibioticoterapia, luego de esto se incluyeron 2 o 3 dilataciones endoscópicas para manejo de la estenosis anastomótica que todos presentaron. La población con ascenso gástrico mediastínico posterior y anastomosis cervical presentó un $43 \%$ de manifestación CD I-II (3 pacientes) y un $57 \%$ de CD III-V (4 pacientes). En este último grupo todos requirieron al menos un aseo quirúrgico y drenaje videotoracoscópico (VTC) y en 2 se empleó una prótesis endoscópica complementaria. La tasa de reoperaciones fue de un $11,7 \%$, siendo todas observadas en pacientes con ascensos vía mediastino posterior; de estas fueron 3 aseos videotoracoscópicos y una reparación de vena innominada. No existió diferencia estadística entre las vías de ascenso y la tasa de reoperaciones $(\mathrm{p}=0,26)$.

La mortalidad quirúrgica fue de 5,8\% (2/34 casos), concentrándose sólo en la población de ascensos por mediastino posterior con anastomosis cervical. Un paciente de 75 años presentó una fístula esofágica que requirió aseos por VTC, en el último aseo tuvo una lesión de vena innominada con posterior shock mixto y fallecimiento; el segundo paciente de 83 años, cursó con una fístula de difícil manejo asociada a colecciones mediastínicas y empiema pleural, requirió múltiples aseos por VTC sin lograr una recuperación adecuada. Estos 2 pacientes representan una mortalidad de 7,4\% (2/27) de los pacientes con ascenso mediastínico y $12,5 \%$ de los pacientes que filtraron (2/16). Esta mortalidad quirúrgica no presentó diferencia estadísticamente significativa entre la población con o sin filtración $(\mathrm{p}=0,139)$.

La estadía hospitalaria fue de 17 días (8-56) presentando una diferencia estadísticamente signifi- cativa entre la población que presentó filtración con p 0,0009 .

El $100 \%$ de los pacientes fueron R0. La sobrevida global a 2 años fue de $53,6 \%$ con una considerable diferencia entre el grupo de fistulizados $(16,7 \%)$ versus aquellos que no $(76,9 \%),(\mathrm{p}=0,003)$ (Figura 2).

En relación a la terapia adyuvante, del 64,7\% de nuestra población que tenía indicación de quimioradioterapia según la decisión del comité oncológico, la mediana de días para inicio de la adyuvancia posterior a la cirugía fue 63 días (47-240 días). Al estudiar los pacientes que presentaron filtración, en la población de filtraciones cervicales, 3 recibieron neoadyuvancia, mientras que en las filtraciones intratorácicas sólo 1. Los esquemas empleados fueron diversos, incluyendo FOLFOX, Xelox, ECF, EOX. En el análisis estadístico la adyuvancia no influyó de manera estadísticamente significativa en las diferencias observadas entre poblaciones con o sin filtración ni relación con el pronóstico.

\section{Discusión}

La fístula de la esofagogastro anastomosis es un evento temido por los cirujanos, dado la morbimortalidad asociada, distintos aspectos de esta entidad explican dicho sentimiento. Existen factores generales y locales asociados a mayor riesgo de fístula los cuales se resumen en:

Sistémicos: Desnutrición, hipovolemia, insuficiencia cardiaca, falla renal, cardiopatía coronaria, vasculopatías, corticoterapia, diabetes mellitus, tabaquismo y quimioterapia sistémica.

Locales: Aporte sanguíneo arterial, estasia venosa, trauma gástrico/inflamación, compresiones extrín27 días (14-50) versus aquella con 11 días (8-56), 
secas, distención gástrica, infecciones, radioterapia.

Técnicos: Tensión, localización anastomosis, técnica anastomótica, refuerzo anastomótico, sutura manual o mecánica.

En nuestra serie hemos observado una alta tasa de filtraciones de la anastomosis cervical, igual a lo señalado en la literatura internacional y las razones de esta mayor tasa de filtración se deben a la longitud del tubo gástrico, probable mayor tensión y déficit de flujo sanguíneo. Otra razón se debe a que, en muchos de nuestros pacientes, hemos efectuado anastomosis manual invaginante que determina cierto grado de obstrucción al flujo de salida hacia distal al tubo gástrico, favoreciendo de esta manera el escape de contenido esofágico hacia el cuello. En los últimos pacientes hemos podido efectuar anastomosis con sutura mecánica con lo cual hemos observado han disminuido las filtraciones.

Varios de estos factores están en muchos de nuestros pacientes, los cuales presentan enfermedades asociadas y también variables netamente de orden técnico. En este artículo hemos analizado algunas variables. Los resultados de este estudio sugieren que: 1. Nuestra población analizada tiende a ser obesa a diferencia de la clásica forma de presentación del cáncer esofágico en nuestro país.

2. La tasa de fístulas esofágicas tiende a ser mayor en ascensos retroesternales, aunque no alcanza la significancia estadística.

3. El tratamiento de las fístulas esofágicas, tras un ascenso retro esternal, es conservador con curaciones, mientras que el enfrentamiento de las fístulas luego de un ascenso trans-mediastínico posterior requiere de reintervenciones y manejo multidisciplinario.

4. El ascenso retroesternal con anastomosis cervical posee mayor seguridad quirúrgica en comparación al ascenso mediastínico posterior en términos de mortalidad operatoria, no así en términos de tasa de filtración.

5. La presencia de fístula anastomótica afecta negativamente la sobrevida global de estos enfermos alcanzando una significancia estadística.

En nuestra institución, en 1995, se realizó la primera esofagectomía videotoraco asistida tipo McKeown, desde entonces, la transición a la vía totalmente mini invasiva ha sido progresiva, es así como en el año 2000 se realizó la primera esofagectomía totalmente mini invasiva con anastomosis cervical ${ }^{5-7}$.

La tasa de complicaciones quirúrgicas observada en este grupo de pacientes con cirugía mínimamente invasiva, es similar a lo observado por nuestro mismo grupo en la era de la cirugía abierta ${ }^{8}$. Sin embargo, la tasa global de morbimortalidad de la esofagectomía ha disminuido significativamente desde la introducción de la esofagectomía asistida por VTC y, posteriormente, la cirugía completamente mini invasiva, junto con la modernización de las unidades de apoyo como unidades de paciente crítico, nutrición intensiva y kinesiología 9 .

Es así como Martin describe una reducción de mortalidad desde $11 \%$ a $2,5 \%(\mathrm{p}<0,001)$ y de mortalidad asociada a fístula desde $43 \%$ a $3,3 \%$ $(\mathrm{p}=0,016)^{1}$. En la experiencia nacional, nuestro centro publicó en 2016 una revisión de cirugía mini invasiva documentándose una mortalidad de 2,8\%, comparable a estándares internacionales ${ }^{2}$. En ese trabajo que incluye 69 pacientes y corresponde a un período de estudio mayor, la incidencia de fístulas cervicales con anastomosis manuales fue mayor $(63,7 \%)$, pero en su mayoría $(60,8 \%)$ fueron fístulas con una clasificación Clavien-Dindo I-II sin ninguna repercusión clínica y mortalidad muy baja. Respecto a sobrevida alejada, los datos obtenidos en la presente publicación son discretamente mejores a nuestros resultados históricos reportados previamente en el año 2000, cuando se analizó el período desde 1985 a 1996, reportándose una sobrevida de $45 \%$ aproximadamente a 2 años de seguimiento ${ }^{10}$.

La frecuencia de las fístulas esofágicas también ha variado en el tiempo, aproximándose a $30 \%$ en $1995^{11}$ y $10 \%$ en $2013^{12}$. Esto puede deberse no sólo a la experiencia de los grupos quirúrgicos, técnica quirúrgica y la vía de abordaje, sino también a los materiales empleados como las suturas reabsorbibles modernas o incluso las suturas mecánicas. Venturelli publica una reducción de $31,3 \%$ a $25 \%$ de fístulas anastomóticas en cánceres esofágicos tratados con intención curativa con abordajes transhiatales y transtorácicos con anastomosis manuales empleando tubo gástrico ${ }^{13,14}$. Este mismo hallazgo se observa en la publicación de nuestro grupo ${ }^{2}$.

Un aspecto a considerar es la acuciosidad del diagnóstico de fístula esofágica (clínico, azul de metileno, radiológico y/o endoscópico), la postura de nuestro equipo es siempre realizar un esofagograma con contraste hidrosoluble entre el $5^{\circ}$ y $7^{\circ}$ día postoperatorio independiente de la evolución, además, ante una mínima sospecha de complicación, monitorizada a través de signos vitales, curva de PCR (tomados a los días 0-3-5 postoperatorios), complementamos con un estudio radiológico y/o endoscópico hasta descartar o confirmar la complicación. Con este método presentamos una tasa de fístula esofágica media de $63,7 \%$, de las cuales $60,8 \%$ clasifican como fístulas menores de manejo conservador, nuestra tasa de reoperaciones es de $13 \%{ }^{2}$. 
El pronóstico del paciente después de una filtración esofágica tanto en mortalidad quirúrgica como en sobrevida global alejada se ve mermado ${ }^{15}$, lo cual es confirmado por nuestra experiencia al analizar las curvas de Kaplan-Meier. Esto posee distintas explicaciones, como:

- El aumento de complicaciones sépticas quirúrgicas tales como infecciones de herida operatoria cervical, mediastinitis, empiema pleural e inclusive peritonitis ${ }^{16,17}$.

- Prolongación de la hospitalización con los subsecuentes eventos adversos médicos no quirúrgicos asociados como neumonías, arritmias, infecciones urinarias, etc. ${ }^{16,17}$.

- Retardo en el inicio de esquemas adyuvantes.

Si bien nuestro estudio no logró identificar factores que predigan de forma estadísticamente significativa la filtración, tampoco pudimos explicar matemáticamente la razón de la diferencia en la sobrevida alejada entre paciente con o sin filtración, esto podría estar explicado por el tamaño de la muestra de nuestra serie.

Asumiendo la gravedad de las fístulas anastomóticas esofágicas se han explorado factores predictivos de esta complicación. Gockel ${ }^{3}$, describió una serie de factores que bajo análisis multivariado determinasen un mayor riesgo de morbilidad y mortalidad después de una esofagectomía para el tratamiento de cáncer esofágico, estos son: tipo de esofagectomía ( $\mathrm{p}=0,0004)$, localización tumoral $(\mathrm{p}=0,02)$, necesidad de transfusión de hemoderivados $(p=0,04)$, edad del paciente $(p=0,003)$, estado nutricional $(\mathrm{p}=0,02)$ y clasificación ASA $(\mathrm{p}=0,04)$. En nuestro trabajo no logramos demostrar significancia estadística en los distintos factores predictivos estudiados. Sin embargo, la edad mostró una tendencia estadística cercana a la significancia.

La clasificación ASA es una herramienta predictiva de morbilidad postoperatoria potente que debe ser considerada; ya en 1988 se definió un incremento significativo en morbilidad postoperatoria, identificando un incremento del riesgo de complicaciones postoperatorias 23 veces mayor para pacientes ASA III en comparación a los ASA $I^{18}$.

Si comparamos las anastomosis cervicales con las mediastínicas, entre el muñón esofágico distal y el tubo gástrico, veremos que las anastomosis cervicales poseen:

- Mayor tasa de fístula anastomótica, alcanzado 5 veces más de riesgo ${ }^{19}$.

- Mayor estenosis y necesidad de dilataciones (50\% de las fístulas desarrollarán estenosis futuras). Las estenosis cervicales requieren 4 dilataciones y las mediastínicas $1^{20}$.
- Mayor tasa de lesión de nervio laríngeo recurrente. Las anastomosis cervicales tienen un 8 a $14 \%$ de paresia de cuerda vocal vs un $0-1 \%$ en las anastomosis intratorácicas ${ }^{20}$.

- Menor morbilidad y cuando se presenta, conlleva consecuencias clínicas leves ${ }^{19,21}$.

- Menor mortalidad dependiente de fístula esofágica, la cual reporta un $5 \%$ en comparación a la anastomosis intratorácica con $12 \%$. Se ha determinado que hasta $71 \%$ de los fallecidos en esofagectomías pueden corresponder a pacientes con anastomosis intratorácicas, versus las cervicales que representan el $6,5 \%{ }^{32}$

- La anastomosis cervical con ascenso mediastínico posterior tiene el riesgo que el débito de la fístula migre hacia caudal precipitando una mediastinitis y sepsis concomitante. Si se usa esta vía debe tenerse la precaución de cerrar el opérculo torácico con puntos entre el tubo gástrico, la fascia prevertebral y los músculos infrahioideos, de esta manera se disminuye el riesgo de mediastinitis.

Dentro de las anastomosis cervicales si discriminamos la vía de ascenso retroesternal de la mediastínica posterior, podremos evidenciar una mayor tasa de filtraciones en las retroesternales. Refrendando este argumento, Orringer publicó en 2001 una serie con más de 1.000 esofagectomías describiendo una tasa de fístulas esofágicas de $13 \%$ en ascensos mediastínicos posteriores y $86 \%$ en ascensos retroesternales ${ }^{23}$. Esta realidad se ha atribuido a un trayecto más largo, lo que confiere una mayor tensión a la anastomosis, si se considera que el extremo proximal del tubo gástrico posee irrigación preferentemente a partir de la red submucosa, la tensión causaría isquemia a una irrigación terminal, lo que afectaría la cicatrización anastomótica.

Respecto a la técnica para realizar la anastomosis existen 2 metaanálisis que describen una menor tasa de filtraciones en sutura mecánica versus manual, sin diferencia estadística entre suturas mecánicas lineales o circulares ${ }^{24,25}$.

En la Tabla 2 se sintetiza parte de la evidencia nacional e internacional referente a esofagectomía.

Tomando en consideración la evidencia internacional y los resultados del presente estudio, recomendamos definir algunas directrices según el escenario clínico:

- En pacientes menores de 65 años, sin grandes comorbilidades, ASA 1-2. El lugar de la anastomosis y el trayecto del tubo gástrico puede realizarse indistintamente siempre que se conserven los estándares oncológicos de la cirugía.

- En pacientes mayores de 65 años, con impor- 
Tabla 2. Evidencia nacional e internacional referente a esofagectomía

\begin{tabular}{|c|c|c|c|c|c|c|c|c|}
\hline Autor & Año & País & $\mathbf{n}$ & Ascenso & Anastomosis & $\begin{array}{c}\text { Fístulas } \\
\%\end{array}$ & $\begin{array}{c}\text { Mortalidad } \\
\%\end{array}$ & $\underset{\%}{\mathrm{SVg}}$ \\
\hline Orringer $^{23}$ & 2001 & USA & 1.085 & Mediastino & Cervical & 13 & 4 & NR \\
\hline Hulscher $^{30}$ & 2002 & USA & 220 & Mediastino & Cervical & 14 & 4 & $40 \%$ \\
\hline Venturelli ${ }^{13}$ & 2003 & Chile & 51 & $\begin{array}{c}\text { Mediastino } \\
\text { Retroesternal }\end{array}$ & Cervical & 23,5 & 6,2 & 29 \\
\hline Martin $^{1}$ & 2005 & USA & 621 & Mediastino & Intratorácica & 6,3 & 2,5 & $30 \%$ \\
\hline Gockel $^{3}$ & 2005 & Alemania & 424 & $\begin{array}{c}\text { Mediastino } \\
\text { Retroesternal }\end{array}$ & Cervical & 18,2 & 6,7 & NR \\
\hline Díaz de Liaño $^{22}$ & 2011 & España & 41 & Mediastínico & Intratorácica & 7,3 & $7,3 \%$ & $27 \%$ \\
\hline Luketich $^{26}$ & 2012 & USA & 1.011 & Mediastino & $\begin{array}{c}\text { Cervical } \\
\text { Intratorácica }\end{array}$ & 5 & 1,68 & NR \\
\hline Klink $^{19}$ & 2012 & Alemania & 72 & Mediastino & $\begin{array}{c}\text { Cervical } \\
\text { Intratorácica }\end{array}$ & $\begin{array}{l}31 \\
11\end{array}$ & 6 & NR \\
\hline Kassis $^{12}$ & 2013 & USA & 7.595 & $\begin{array}{c}\text { Mediastino } \\
\text { Retroesternal }\end{array}$ & $\begin{array}{c}\text { Cervical } \\
\text { Intratorácica }\end{array}$ & $\begin{array}{c}12,3 \\
9,3\end{array}$ & $\begin{array}{c}4,4 \\
11,6\end{array}$ & NR \\
\hline Makar $^{31}$ & 2013 & UK & 1.108 & $\begin{array}{l}\text { Mediastino } \\
\text { Retroesternal }\end{array}$ & $\begin{array}{c}\text { Cervical } \\
\text { Intratorácica }\end{array}$ & $\begin{array}{c}13,6 \\
2,9\end{array}$ & NR & NR \\
\hline $\mathrm{Mao}^{28}$ & 2015 & China & 59 & Mediastino & Cervical & 23,7 & 0 & NR \\
\hline van Rossum ${ }^{15}$ & 2017 & Holanda & 286 & Mediastino & Cervical & 21 & $\begin{array}{l}4 \text { Sin mediastinitis } \\
14 \text { Con mediastinitis }\end{array}$ & 40 \\
\hline Braghetto & 2017 & Chile & 34 & $\begin{array}{c}\text { Mediastino } \\
\text { Retroesternal }\end{array}$ & $\begin{array}{c}\text { Cervical } \\
\text { Intratorácica }\end{array}$ & $\begin{array}{l}38,2 \\
14,2\end{array}$ & $\begin{array}{c}5,8 * \text { Con mediatinitis } \\
0 \text { Sin mediastinitis }\end{array}$ & 53,6 \\
\hline
\end{tabular}

NR: No reportado. *Mortalidad observada solo en los pacientes con anastomosis cervical y ascenso mediastínico.

tantes comorbilidades, ASA 3-4, desnutridos. Se sugiere emplear la anastomosis cervical con ascenso retroesternal, dado la menor reserva funcional de estos pacientes y el riesgo de mortalidad conferido por la potencial fístula. Sin embargo, esto debe ser adecuado a las preferencias de cada cirujano.

\section{Conclusiones}

Las filtraciones en anastomosis esofágicas son frecuentes en pacientes operados con intención curativa de cáncer esofágico. Las fístulas con ascenso retroesternal presentan un comportamiento más benigno sin mortalidad operatoria, mientras que el ascenso por mediastino posterior concentró las morbilidades mayores y la mortalidad quirúrgica. Las fístulas esofágicas afectan negativamente la sobrevida global de los pacientes con esofagectomía oncológica.

\section{Responsabilidades éticas}

Protección de personas y animales. Los autores declaran que para esta investigación no se han realizado experimentos en seres humanos ni en animales.

Confidencialidad de los datos. Los autores declaran que en este artículo no aparecen datos de pacientes.

Derecho a la privacidad y consentimiento informado. Los autores declaran que en este artículo no aparecen datos de pacientes.

\section{Financiación}

El presente trabajo no posee fuente de financiación.

\section{Conflicto de intereses}

Los autores declaran no tener ningún conflicto de intereses. 


\section{Bibliografía}

1. Martin LW, Swisher SG, Hofstetter W, Correa AM, Mehran RJ, Rice DC, Vaporciyan AA, et al. Intrathoracic leaks following esophagectomy are no longer associated with increased mortality. Ann Surg. 2005;242:392-402.

2. Braghetto I, Cardemil G, Csendes A, Lanzarini E, Mushle M, Venturelli F, et al. Resultados de la cirugía actual para el tratamiento del cáncer de esófago. Rev Chil Cir. 2016;68:94-106

3. Gockel I, Exner C, Junginger T. Morbidity and mortality after esophagectomy for esophageal carcinoma: A risk analysis. World J Surg Oncol. 2005;3:37.

4. Jones CE, Watson TJ. Anastomotic Leakage Following Esophagectomy. Thorac Surg Clin. 2015;25:449-59.

5. Braghetto I, Korn O, Burdiles P. Surg Laparosc Endosc Percutan Tech. 2001;4:119-25.

6. Braghetto I, Csendes A, Cardemil G, Burdiles P, Korn O, Valladares H. Open transthoracic or transhiatalesophagectomy versus minimally invasive esophagectomy in terms of morbidity, mortality and survival. Surg Endosc. 2006;20:1681-6.

7. Braghetto I, Csendes A, Rappoport J, Chong H, Debandi A. video assisted transhiatal esophagectomy Video Revista de Cirugia. Video Review of Surgery. 1997;14:25-32.

8. Braghetto I, Csendes A, Cardemil G, Burdiles P, Rodríguez A, Guerra J, Complicaciones de la esofagectomía: diagnóstico, mecanismo fisiopatológico, prevención y manejo. Rev Chil Cir. 2002;54:451-63.

9. Ben-David K, Sarosi GA, Cendan JC. Decreasing morbidity and mortality in 100 consecutive minimally invasive esophagectomies. Surg Endosc. 2012;26:162-7.

10. Braghetto I, Csendes A, Cornejo A, Cardemil G. Sobrevida de pacientes con cáncer de esófago sometidos a esofagectomía total torácica. Rev Med Chile 2000;128:64-74.

11. Urschel JD. Esophagogastrostomy anastomotic leaks complicating esophagectomy: a review. Am J Surg. 1995;169:634-40.

12. Kassis ES, Kosinski AS, Ross P Jr.
Predictors of anastomotic leak after esophagectomy: an analysis of the Society of Thoracic Surgeons General Thoracic Database. Ann Thorac Surg. 2013;96:1919-26.

13. Venturelli A, Soto S, Díaz J, Cardemil B, Sánchez A, Jiménez L. Cáncer de esófago, tratamiento en el Hospital Clínico Regional de Valdivia durante el período 1982-2001. Rev Chil Cir. 2003;55:361-84.

14. Venturelli A, Sánchez A, Cardemil B, Díaz J, Jiménez J, Cárcamo C. Cáncer de esófago. Sobrevida a 10 años plazo. Rev Chil Cir. 2001;53:541-4.

15. van Rossum PS, Haverkamp L, Carvello M, Ruurda JP, van Hillegersberg R. Management and outcome of cervical versus intrathoracic manifestation of cervical anastomotic leakage after transthoracic esophagectomy for cancer. Dis Esophagus. 2017;30:1-8.

16. Conners RC, Reuben BC, Neumayer LA. Comparing outcomes after transthoracic and transhiatal esophagectomy: a 5-year prospective cohort of 17,395 patients. J Am Coll Surg. 2007;205:735-40.

17. Tabatabai A, Hashemi M, Mohajeri G Incidence and risk factors predisposing anastomotic leak after transhiatal esophagectomy. Ann Thorac Med. 2009;4:197-200

18. Tiret L, Hatton F, Desmonts JM, Vourc'h G. Prediction of outcome of anaesthesia in patients over 40 years: A multifactorial risk index. Stat Med. 1988;7:947-54

19. Klink CD, Binneb€osel M, Otto J, Boehm $\mathrm{G}$, von Trotha KT, Hilgers RD, et al. Intrathoracic versus cervical anastomosis after resection of esophageal cancer: a matched pair analysis of 72 patients in a single center study. World J Surg Oncol. 2012;10:159

20. van Workum $F$, van der Maas J, van den Wildenberg FJ, Polat F, Kouwenhoven EA, van Det MJ, et al. Improved Functional Results After Minimally Invasive Esophagectomy: Intrathoracic Versus Cervical Anastomosis. Ann Thorac Surg. 2017;103: 267-73.

21. Sarli D, Bona D, Abraham M, Bonavina L. Conservative and surgical treatment of esophago-gastric anastomotic leaks. Ann Ital Chir 2006;77:391-6.

22. Díaz de Liaño A, Sánchez G, Yárnoz
C, Artajona A. Oesophagogastric anastomosis complications in the Ivor Lewis operation. Cir Esp. 2011;89:175-81.

23. Orringer MB, Marshall B, Iannettoni MD. Transhiatal esophagectomy for treatment of benign and malignant disease. World J Surg. 2001;25:196-203.

24. Deng XF, Liu QX, Zhou D. Hand-sewn vs linearly stapled esophagogastric anastomosis for esophageal cancer: a meta-analysis. World J Gastroenterol. 2015;21:4757-64.

25. Zhou D, Liu QX, Deng XF. Comparison of two different mechanical esophagogastric anastomosis in esophageal cancer patients: a metaanalysis. J Cardiothorac Surg. 2015; 10:67.

26. Luketich JD, Pennathur A, Awais O, Levy RM, Keeley S, Christie NA, et al. Outcomes After Minimally Invasive Esophagectomy. Review of Over 1000 Patients. Ann Surg. 2012;256:95-103.

27. Wright CD, Kucharczuk JC, O'Brien SM, Grab JD, Allen MS. Predictors of major morbidity and mortality after esophagectomy for esophageal cancer: a Society of Thoracic Surgeons General Thoracic Surgery Database risk adjustment model. J Thorac Cardiovasc Surg. 2009;137:587-95.

28. Mao T, Fang W, Gu Z, Guo X, Ji C, Chen W. Comparison of perioperative outcomes between open and minimally invasive esophagectomy for esophageal cancer. Thorac Cancer 2015;6:303-6.

29. Jones CE. Watson A. Anastomotic leakage following esophagectomy. Thorac Surg Clin. 2015;25:449-59.

30. Hulscher JB, van Sandick JW, de Boer AG. Extended trasnthoracic resection compared to limited transhiatal resection for adenocarcinoma of the esophagus. $\mathrm{N}$ Engl J Med. 2002;347:1662-9.

31. Markar SR, Arya S, Karthikesalingam A. Technical factors that affect anastomotic integrity following esophagectomy: systematic review and meta-analysis. Ann Surg Oncol. 2013;20:4274-81.

32. Sauvante A, Mariette $C$, Thomas $P$, Lozac'h P, Segol P, Triet E, et al. Mortality and morbidity after resection of adenocarcinoma of the gastroesophageal junction: predictive factors. J Am Coll Surg. 2005;201:253-62. 